\title{
Three-hadron angular correlations from perturbative QCD at the Relativistic Heavy lon Collider and at the Large Hadron Collider
}

\author{
Alejandro Ayala, Isabel Dominguez \\ Instituto de Ciencias Nucleares, Universidad Nacional Autónoma de México, Apartado Postal \\ 70-543, México Distrito Federal 04510, Mexico. \\ E-mail: ayala@nucleares.unam.mx, isabel@nucleares.unam.mx
}

Jamal Jalilian-Marian

Department of Natural Sciences, Baruch College, New York, New York 10010, USA and CUNY Graduate Center, 365 Fifth Avenue, New York, New York 10016, USA.

E-mail: jamal.jalilian-marian@baruch. cuny.edu

\section{Javier Magnin}

Centro Brasileiro de Pesquisas Físicas, CBPF, Rua Dr. Xavier Sigaud 150, 22290-180, Rio de Janeiro, Brazil.

E-mail: jmagnin@cbpf.br

\section{Maria Elena Tejeda-Yeomans ${ }^{* \dagger}$}

Departamento de Física, Universidad de Sonora, Boulevard Luis Encinas J. y Rosales, Colonia Centro, Hermosillo, Sonora 83000, Mexico.

E-mail: elena.tejeda@correo.fisica.uson.mx

\begin{abstract}
We study three-hadron azimuthal angular correlations in high energy proton-proton and central nucleus-nucleus collisions at RHIC and LHC at mid-rapidity. We use the lowest order parton matrix elements for 2 to 3 processes and include the effect of parton energy loss in the quark-gluon plasma using the modified fragmentation function approach. For the case when the produced hadrons have comparable momenta, we observe two away side peaks at $2 \pi / 3$ and $4 \pi / 3$. We consider the dependence of the angular correlations with energy loss parameters that have been used in studies of single inclusive hadron production at RHIC. Our results on the angular dependence of the cross section agree well with the experimental data measured by the PHENIX collaboration. We comment on the possible contribution of 2 to 3 processes to di-hadron angular correlations and how a comparison of the two processes may help to further characterize the plasma.
\end{abstract}

The Seventh Workshop on Particle Correlations and Femtoscopy

September 20th to 24th, 2011

The University of Tokyo, Japan

\footnotetext{
* Speaker.

${ }^{\dagger}$ Support to participate in this event has been received in part by CONACyT (Mexico) under grant number 128534, PAPIIT-UNAM under grant number IN103811, by DCEN and DIP at the Universidad de Sonora
} 


\section{Introduction}

Some of the most remarkable evidence of the Quark-Gluon Plasma (QGP) influence on the propagation of a parton within it has emerged from data taken at the Relativistic Heavy Ion Collider (RHIC) and the Large Hadron Collider (LHC) from colliding nuclei experiments. The parton that traverses the medium losing its energy, does so in such a way that may explain for example: the observed suppression of single hadron transverse momentum spectra in $\mathrm{Au}+\mathrm{Au}$ collisions, as compared to $\mathrm{p}+\mathrm{p}$ collisions and, the disappearance of the away-side peak in two-particle azimuthal correlations [1].

Given the array of energy loss models that are able to reproduce this suppression, it seems logic to pursue the study of energy loss dynamics of these partons in the medium, using more exclusive observables such as di-hadron and three-hadron angular correlations. In particular, the double hump structure on the away-side in azimuthal two-particle correlations in $\mathrm{Au}+\mathrm{Au}$ collisions [2] could be explained via the three-hadron correlations channel. As we have seen during this workshop, these observables have proven to be a powerful tool to point towards the more robust theoretical models $[3,4]$.

In Ref. [5] we showed that some properties of the two-particle correlations can be understood from three-hadron production studies. We considered the different path lengths traveled by the two away side partons in $2 \rightarrow 3$ versus the away side parton in $2 \rightarrow 2$ parton processes. Since the parton that travels the smaller path length in $2 \rightarrow 3$ processes in the away side has on the average, also a smaller path length to go through than the away side parton in $2 \rightarrow 2$ processes, then it has a smaller chance of loosing energy than its counterpart in the $2 \rightarrow 2$ processes. The two effects combined and upon accumulation of signal, look like a double hump or a broader peak in the away side, for certain momentum difference between leading and associate particles.

Our latest efforts were focused on comparing results emerging from these ideas with preliminary data for the three-hadron correlation function [6]. In this article we present a summary of these results and preliminary results on the follow up of this work.

\section{A puzzle in angular correlation studies}

It has been repeatedly reported that there is a rich correlation structure found in $\mathrm{Au}+\mathrm{Au}$ collision studies when compared to similar $\mathrm{p}+\mathrm{p}$ collision studies. In particular the ridge and broad away side structures are so named after an observation of an excess yield of correlated particles at $\Delta \phi=0^{\circ}$ and $\Delta \phi \approx 120^{\circ}$ extending out to $\Delta \eta>2$ as can be seen in the head $v s$ shoulder analysis of FIG.6 and 7 in Ref [7]. There we can see that the head/shoulder ratio $R_{H S}$, grows with $p_{T}$ for $\mathrm{p}+\mathrm{p}$ collisions which is correlated with the fact that at those scales the jet gets narrower. At the same time, we observe that for $p_{T}$ smaller than $4 \mathrm{GeV}$ in $\mathrm{Au}+\mathrm{Au}$ collisions, the medium effects dominate the signal while jet fragmentation dominates at high $p_{T}$. So, below $3-4 \mathrm{GeV}$ we expect to find the origins of the double-hump structure by having a clear understanding and implementation of energy loss dynamics for the hard partons that are propagating in the medium.

In the past, several theoretical models have been used to look for the origins of these structures, such as back splash, momentum kick, glasma flux tubes, Mach cone shock waves, transverse radial flow, parton recombination and more recently, initial state fluctuations [8]. In these proceedings 


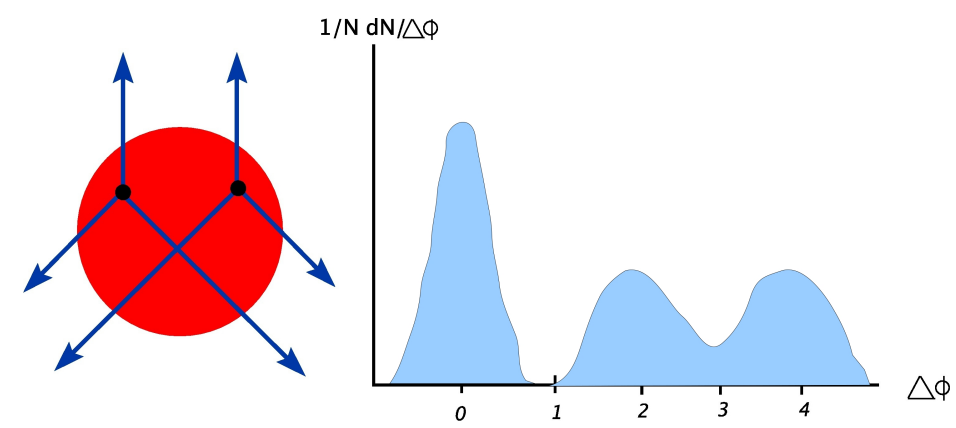

Figure 1: Accumulated signal for $2 \rightarrow 3$ processes (that get enhanced with respect of the $2 \rightarrow 2$ away side peak at $\pi$ ) where one parton in the away side cannot emerge from the medium, to account for a double hump structure in the away side of three-hadron azimuthal correlation studies.

P.K. Netrakanti from the STAR collaboration discusses many of these models, their successes and drawbacks. Most of these theoretical models rely heavily on the description of ridges and humps as macroscopical manifestation of collective behavior but, none of them has a stern handle on all the special characteristics of these structures on both pseudorapidity and angle variations.

\section{Our proposal, in a nutshell}

We consider the three-hadron production cross sections in $\mathrm{p}+\mathrm{p}$ and $\mathrm{A}+\mathrm{A}$ collisions using leading order $2 \rightarrow 3$ partonic processes. We include the QGP effect by parton energy loss on the final particle spectra, using medium modified fragmentation functions. Then we investigate the effect of energy loss on the angular correlations of the three produced hadrons by varying the parameters associated with the energy loss suffered by each parton. Finally, we compare our results to the preliminary data on the three-hadron correlation function measured at RHIC [6]. Moreover, we press on to have a Monte Carlo event generator, to be able to widen our analysis with less restrictive kinematical cuts. Some preliminary results are shown regarding our latest advances.

As we mentioned before, the main idea we want to investigate is sketched in Figure 1: can the accumulation of signal due to the effects of $2 \rightarrow 2$ vs $2 \rightarrow 3$ away side partons missing together with energy loss in the medium, account for a double hump in the away side of angular correlations?

In order to obtain the three hadron azimuthal correlation, we use the full $2 \rightarrow 3$ differential cross section

$$
\frac{d \sigma^{p p \rightarrow h 1 h 2 h 3 X}}{d y d h_{\{1,2,3\}} d \Delta \phi} \propto \int d z_{3}\left|\mathscr{M}^{2 \rightarrow 3}\right|^{2} f_{i / p}\left(x_{1}, \mu^{2}\right) f_{j / p}\left(x_{2}, \mu^{2}\right) D_{h 1 / k}^{0}\left(z_{1}, \mu^{2}\right) D_{h 2 / m}^{0}\left(z_{2}, \mu^{2}\right) D_{h 3 / n}^{0}\left(z_{3}, \mu^{2}\right)
$$

where $\mathscr{M}^{2 \rightarrow 2}$ and $\mathscr{M}^{2 \rightarrow 3}$ are the leading order partonic matrix elements and $f_{i / p}$ and $D_{h / k}$ are the distribution and fragmentation functions respectively. The matrix elements at the parton level [9] $\mathscr{M}_{i j \rightarrow \mathrm{P}_{k} \mathrm{P}_{l} \mathrm{P}_{m}}$ are divergent due both to collinear and soft singularities but, kinematic divergences are universal and can be removed using well known techniques and we will make some remarks at the end. For now we apply angular cuts to avoid the divergent regions. We use the CTEQ6 parametrization [10] of the parton distribution functions $f_{i / p}\left(x, \mu^{2}\right)$ and the KKP parametrization [11] of the parton fragmentation functions $D_{h / k}^{0}\left(z, \mu^{2}\right)$ for $\mathrm{p}+\mathrm{p}$ collisions. For $\mathrm{Au}+\mathrm{Au}$ collisions we use 
modified fragmentation functions [12] $D_{h / i}\left(z_{i}, \mu^{2}\right)$ to get the medium induced energy loss effects into the cross sections. Basically, these modified functions arose when the authors of Ref. [12] considered an initial gluon density obtained from the overlap of two colliding nuclei, each with a Woods-Saxon density profile. The gluon density of the medium is diluted only due to the longitudinal expansion of the plasma and transverse expansion is neglected.

As can be seen in Figure 1 of Ref. [5] the $\sqrt{s}=200 \mathrm{GeV}$ differential cross section for $\mathrm{p}+\mathrm{p}$ collisions for the away side hadrons as a function of the azimuthal angle in a midrapidity region $\left(y_{i}=0\right)$ for a $10 \mathrm{GeV} / \mathrm{c}$ hadron momenta symmetrical configuration shows a peak in $\Delta \phi=\pi$ or at $\Delta \phi=2 \pi / 3,4 \pi / 3$ if there is a 2 or a 3 final state hadron configuration. When we compare the same result but from $\mathrm{Au}+\mathrm{Au}$ collisions, we see that there is more than a factor of two enhancement for the $\mathrm{Au}+\mathrm{Au}$ peaks in the away side when comparing 3 with respect to 2 final state events.

The sole ingredient that distinguishes the in-vacuum from the in-medium calculation is the energy loss of partons that hadronize collinearly, so this factor of two effect is perhaps due to the different geometry for the trajectories of 3 as opposed to 2 particles in the final state. In this reference we also tested this idea by direct computation of distribution of path lengths: indeed we found that in $2 \rightarrow 3$ processes, there is a large probability to have one of the two away side particles being absorbed and the other randomly getting out, producing on the average a double hump structure in the azimuthal correlations.

\section{Results}

In Ref. [6] we calculated the azimuthal correlations of the three produced hadrons by varying the parameters associated with the energy loss $\left(\varepsilon_{0}\right.$ characterizes the average energy loss per unit of path traversed) and the center of mass energy for both RHIC and LHC energies. Then we compare our results to preliminary data on the three-hadron correlation function measured at RHIC and we see that the angular dependence of the correlation function is reproduced correctly.

Note that in Figure 2 when hadrons have same or not too different momenta, we observe two peaks on the away side, with respect to the leading hadron, at $\pi / 5 \leq \theta_{2}^{\mathrm{H}}\left(\theta_{3}^{\mathrm{H}}\right) \leq 4 \pi / 5,6 \pi / 5 \leq$ $\theta_{3}^{\mathrm{H}}\left(\theta_{2}^{\mathrm{H}}\right) \leq 9 \pi / 5$. In fact the location of the peaks stays the same and their intensity decreases as the difference between away and leading particle momenta increases whereas the intensity of the peaks drops as we increase the energy loss parameter. In Figure 3 we compare our results with preliminary three-particle correlations from the PHENIX collaboration provided in reference [4]. In order to compare with the data we take $\Delta \phi=\theta_{3}^{\mathrm{H}}-\theta_{2}^{\mathrm{H}}$ and the hadrons with momentum bins at $2.5 \leq h_{1} \leq 4 \mathrm{GeV}, 1 \leq h_{2}, h_{3} \leq 2.5 \mathrm{GeV}$ and integrated over a $\Delta \theta=\theta_{2}^{\mathrm{H}}$ in $1.65 \leq \Delta \theta \leq 2.2 \mathrm{rad}$ for different values of $\varepsilon_{0}=1,2,3 \mathrm{GeV} / \mathrm{fm}$. As you can see the angular region $1.5 \lesssim \Delta \phi \lesssim 2.7$ radians is well described here, particularly for the value of $\varepsilon_{0}=2 \mathrm{GeV} / \mathrm{fm}$.

\section{Conclusions and follow up}

We can summarize what has been discussed as follows: (1) the observation of $2 \rightarrow 3$ processes should be enhanced with respect to $2 \rightarrow 2$ processes in $\mathrm{Au}+\mathrm{Au}$ collisions compared with $\mathrm{p}+\mathrm{p}$ collisions; (2) this effect has a bearing on the away side shape for different kinematical cuts in $\mathrm{Au}$ + Au collisions; (3) our three-hadron correlations show the angular region $1.5 \lesssim \Delta \phi \lesssim 2.7 \mathrm{rad}$ is 

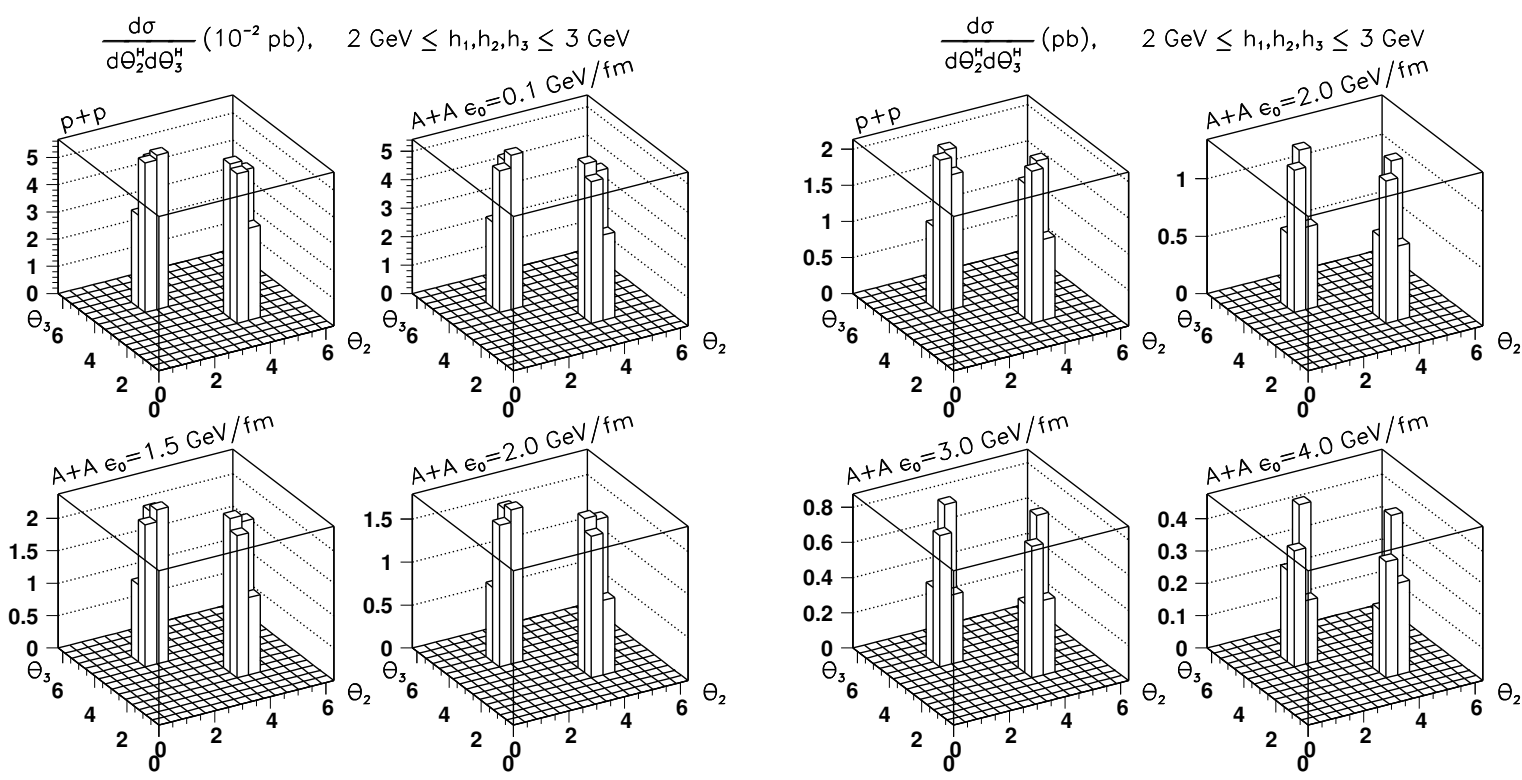

Figure 2: On the left, we show the angular differential cross section for hadron in the $2-3 \mathrm{GeV}$ range and for $\sqrt{s_{N N}}=200 \mathrm{GeV}$ collisions and varying $\varepsilon_{0}=0,0.1,1.5,2 \mathrm{GeV} / \mathrm{fm}$. On the right, we show the corresponding ones for $\sqrt{s_{N N}}=2.7 \mathrm{TeV}$ collisions and varying $\varepsilon_{0}=0,2,3,4 \mathrm{GeV} / \mathrm{fm}$. From Ref. [6] with copyright (2011) by the American Physical Society.

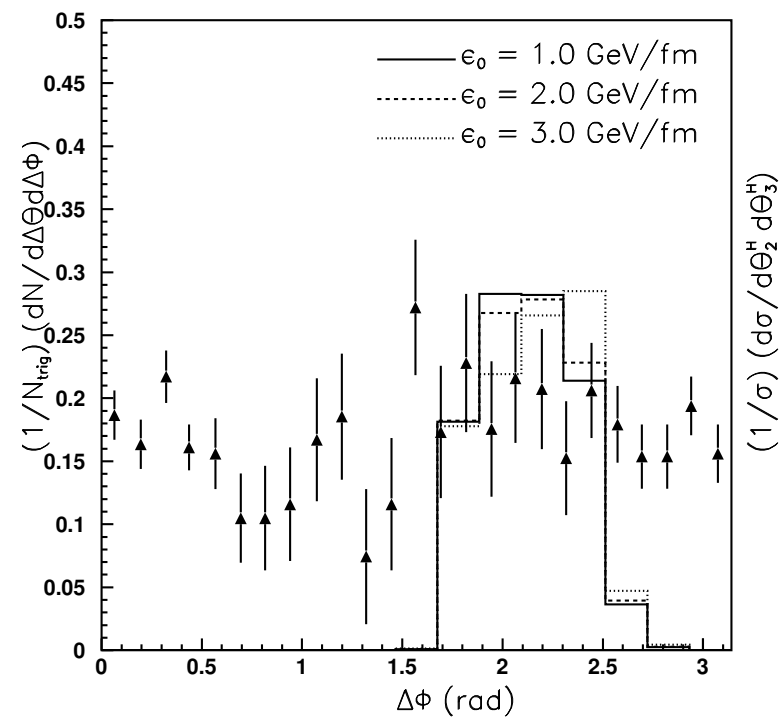

Figure 3: Cross section $d \sigma / d \theta_{2}^{\mathrm{H}} \theta_{3}^{\mathrm{H}}$ as a function of the angular difference $\Delta \phi=\theta_{3}^{\mathrm{H}}-\theta_{2}^{\mathrm{H}}$ for a leading hadron momentum $2.5 \mathrm{GeV} \leq h_{1} \leq 4 \mathrm{GeV}$ and away-side hadron momenta $1 \mathrm{GeV} \leq h_{2}, h_{3} \leq 2.5 \mathrm{GeV}$ integrated over a $\Delta \theta=\theta_{2}^{\mathrm{H}}$ angular range $1.65 \leq \Delta \theta \leq 2.2 \mathrm{rad}$. Note that histograms are normalized to their cross section, $\sigma$, obtained by integration of the differential cross section over the above angular ranges and correspond to three values of the energy loss parameter $\varepsilon_{0}=1,2,3 \mathrm{GeV} / \mathrm{fm}$. The calculation is compared to preliminary data from PHENIX. From Ref. [6] with copyright (2011) by the American Physical Society. 
well described with our model, particularly for the value of $\varepsilon_{0}=2 \mathrm{GeV} / \mathrm{fm}$. Nevertheless, we still have an ongoing investigation to improve our results in several fronts: (i) quantify the contribution of these $2 \rightarrow 3$ processes to di-hadron correlations: via the loss of one parton or via the projection of the three-hadron angular correlation onto two-dimensions (this could happen when either one of the partons travels a large distance in the plasma and loses a large portion of its energy, so that its transverse momentum is outside the transverse momentum window considered for the associate hadrons); (ii) compare these contributions with those of genuine $2 \rightarrow 2$ processes both in $\mathrm{p}+\mathrm{p}$ and in $\mathrm{Au}+\mathrm{Au}$ collisions; (iii) study the dependence on the transverse plane location of these events since it will provide more information on the QGP tomography. In order to further explore these and other questions we are developing a Monte Carlo event generator that uses MadGraph/MadEvent [13] capabilities where higher order matrix elements with an automated version of the dipole subtraction method are already implemented so we can relax some of the angular cuts already used in the results presented above. Results on the use of this event generator will be soon reported elsewhere [14].

\section{References}

[1] S.S. Adler et al. (PHENIX Collaboration), Phys. Rev. Lett. 91, 072301 (2003); J. Admas et al. (STAR Collaboration), Phys. Rev. Lett. 91, 172302 (2003); S.S. Adler et al. (PHENIX Collaboration), Phys. Rev. C 69, 034910 (2004); C. Adler et al. (STAR Collaboration), Phys. Rev. Lett. 90082302 (2003); J. Adams et al. (STAR Collaboration), Nucl. Phys. A 757, 102 (2005); J. Adams et al. (STAR Collaboration), Phys. Rev. Lett. 97, 162301 (2006)

[2] J. Adams et al. (STAR Collaboration), Phys. Rev. Lett. 95, 152301 (2005); A. Adare et al. (PHENIX Collaboration), Phys. Rev. C 77, 011901(R) (2008); A. Adare et al. (PHENIX Collaboration), Phys. Rev. C 78, 014901 (2008).

[3] B.I. Abelev et al. (STAR Collaboration), Phys. Rev. Lett. 102, 052302 (2009).

[4] N.N. Ajitanand (for the PHENIX Collaboration), Indian J. Phys. 84, 1647 (2010).

[5] A. Ayala, J. Jalilian-Marian, J. Magnin, A. Ortiz, G. Paić and M. E. Tejeda-Yeomans, Phys. Rev. Lett. 104, 042301 (2010).

[6] A. Ayala, J. Jalilian-Marian, J. Magnin, A. Ortiz, G. Paić and M. E. Tejeda-Yeomans, Phys. Rev. C 84, 024915 (2011).

[7] A. Adare et al. (PHENIX Collaboration), Phys. Rev. C 78, 014901 (2008)

[8] V. S. Pantuev, arXiv:0710.1882, 2007, C.-Y. Wong, PRC78:064905, 2008, A. Dumitru et al, NPA810:91, 2008, J. Ruppert et al, APPS1:633, 2008, C.A. Pruneau et al, NPA802:107, 2008, R.C. Hwa, arXiv:0904.2159, 2009 B. Alver et al, PRC81:054905, 2010.

[9] R.K. Ellis and J.C. Sexton, Nucl. Phys. B 269, 445 (1986).

[10] H. L. Lai, J. Huston, Z. Li, P. Nadolsky, J. Pumplin, D. Stump and C.P. Yuan, Phys. Rev. D 82, 054021 (2010).

[11] B.A. Kniehl, G. Kreimer and B. Potter, Nucl. Phys. B 582, 514 (2000).

[12] H. Zhang, J.F. Owens, E. Wang and X.-N. Wang, Phys. Rev. Let.98, 212301 (2007).

[13] J. Alwall et al., JHEP 0709, 28 (2007).

[14] A. Ayala, I. Domínguez, J. Jalilian-Marian, J. Magnin and M. E. Tejeda-Yeomans, in progress. 